\title{
Efficacy of a Mucoadhesive Hydrogel (MuGard $\AA$ ) in Patients with high risk of Oral Mucositis during Cytotoxic Chemotherapy: A Multicenter, Double-Blind, Randomized Phase III Trial
}

Song Ee Parka, In Gyu Hwang ${ }^{a}$, Hee Jun Kimª, Jun Ho Yia, Sung Yong Ohb, Jung Hun Kang ${ }^{c}$, Kyung-Hee Lee ${ }^{d}$, Joung-Soon Jang ${ }^{a *}$

aDepartment of Internal Medicine, Chung-Ang University College of Medicine, Seoul, South Korea, bepartment of Internal Medicine, Dong-A University College of Medicine, Busan, South Korea, cDepartment of Internal Medicine, Gyeongsang National University Hospital, Gyeongsang National University School of Medicine, Jinju, South Korea, dDepartment of Internal Medicine, Yeungnam University Medical Center, Daegu, South Korea.

\section{INTRODUCTION}

Oral mucositis is a common, painful side effect of chemotherapy.

The objective of trial was to evaluate the preventive effect of mucoadhesive hydrogel (MuGard®) on the development of oral mucositis in the patients who had oral mucositis during previous chemotherapy.

\section{STUDYDESIGN}

In conventional multi-institutional, double-blind, randomized, placebo-controlled group, phase III study, we enrolled patients who had a World Health Organization (WHO) grade 2 or higher oral mucositis in previous cycle chemotherapy were randomized to receive either MuGard or saline during chemotherapy.

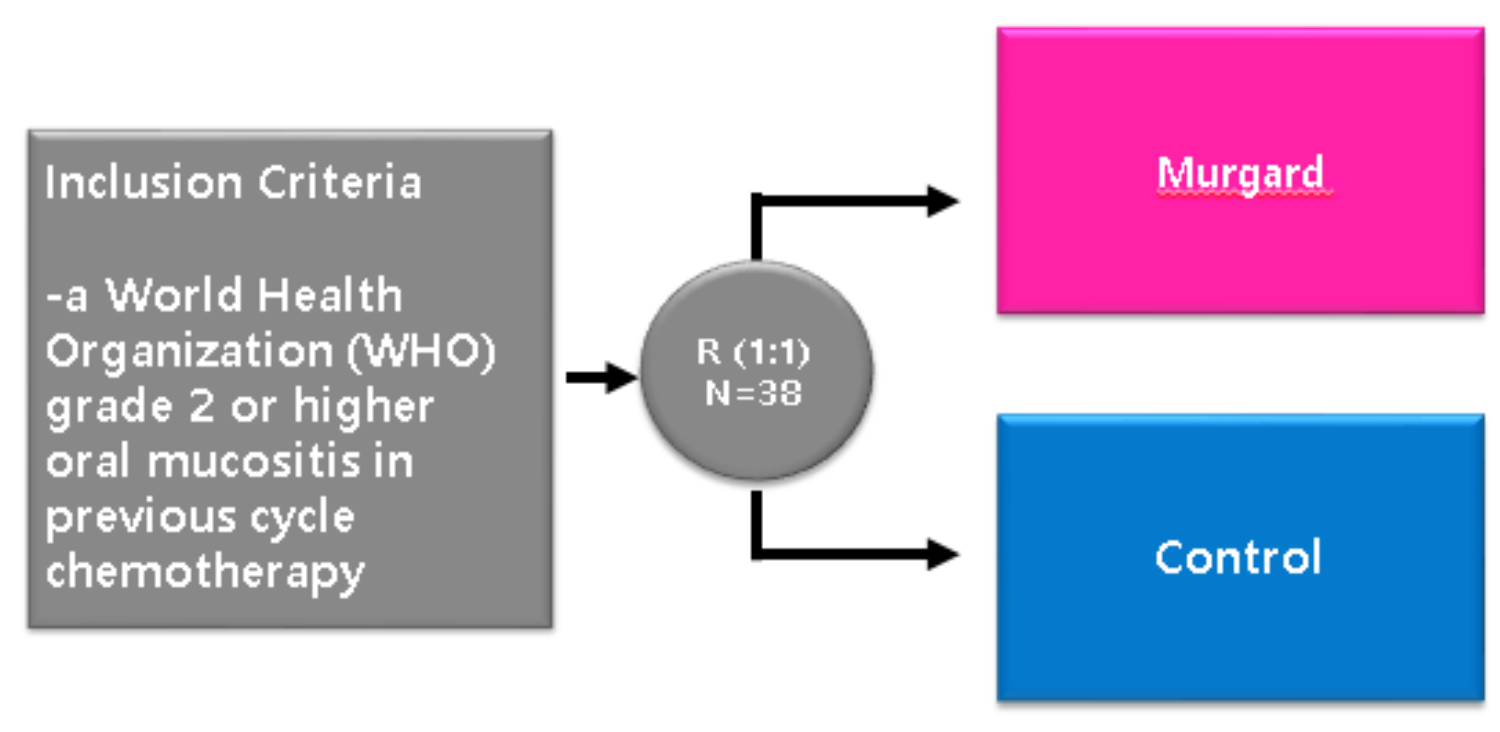

< Inclusion criteria>

1. ECOG $\leq 2 ; 18<$ age $<70$

2. World Health Organization (WHO) grade 2 or higher oral mucositis in previous cycle chemotherapy

\section{$<$ Primary Endpoint>}

- A reduction in mouth and throat soreness (MTS)

MTS was defined by area under the curve (AUC) of the OMDQ MTS question2 (Q2) score.

\section{RESULTS}

TABLE 1. Characteristics of patients $(\mathrm{N}=38)$

\begin{tabular}{lccc}
\hline Characteristic & $\begin{array}{r}\text { Control } \\
(\mathrm{N}=18)\end{array}$ & $\begin{array}{r}\text { Mugard } \\
(\mathrm{N}=20)\end{array}$ & P value \\
\hline Age , year & $66(55-80)$ & $61(49-84)$ & 0.114 \\
$\quad$ Median (range) & $6(33 \%)$ & $12(60 \%)$ & 0.100 \\
Sex & $12(67 \%)$ & $8(40 \%)$ & \\
$\quad$ Male & & & \\
Female & $2(11 \%)$ & $1(5 \%)$ & 0.403 \\
Location of primary tumor & $1(6 \%)$ & $2(10 \%)$ & \\
Breast cancer & $7(38 \%)$ & $5(25 \%)$ & \\
Stomach cancer & $1(6 \%)$ & $4(20 \%)$ & \\
Colon caner & $2(11 \%)$ & $4(20 \%)$ & \\
Rectal cancer & $1(6 \%)$ & $3(15 \%)$ & \\
Lung cancer & $4(22 \%)$ & $1(5 \%)$ & \\
Lymphoma & & & \\
Other &
\end{tabular}

\begin{tabular}{lccc}
\hline Characteristic & $\begin{array}{c}\text { Control } \\
(\mathrm{N}=18)\end{array}$ & $\begin{array}{c}\text { Mugard } \\
(\mathrm{N}=20)\end{array}$ & P value \\
\hline Prior oral mucositis score - WHO scale & & \\
Grade 2 & $12(67 \%)$ & $9(45 \%)$ & 0.180 \\
Grade 3 & $6(33 \%)$ & $11(55 \%)$ & \\
AJCC stage & & & \\
I & $1(6 \%)$ & $0(0 \%)$ & 0.278 \\
II & $1(6 \%)$ & $4(20 \%)$ & \\
III & $1(6 \%)$ & $3(15 \%)$ & \\
IV & $15(82 \%)$ & $13(65 \%)$ & \\
ECOG & & & \\
0 & $1(6 \%)$ & $2(10 \%)$ & 0.878 \\
1 & $15(82 \%)$ & $16(80 \%)$ & \\
2 & $2(12 \%)$ & $2(10 \%)$ & \\
prior radiation therpay & & & 0.544 \\
No & $15(82 \%)$ & $14(70 \%)$ & \\
Yes (head and neck area) & $1(6 \%)$ & $1(5 \%)$ & \\
Yes (Other area) & $2(12 \%)$ & $5(25 \%)$ & \\
\hline
\end{tabular}

Figure 1. Relation between mean Oral Mucositis Daily Questionnaire between control and mugard groups.

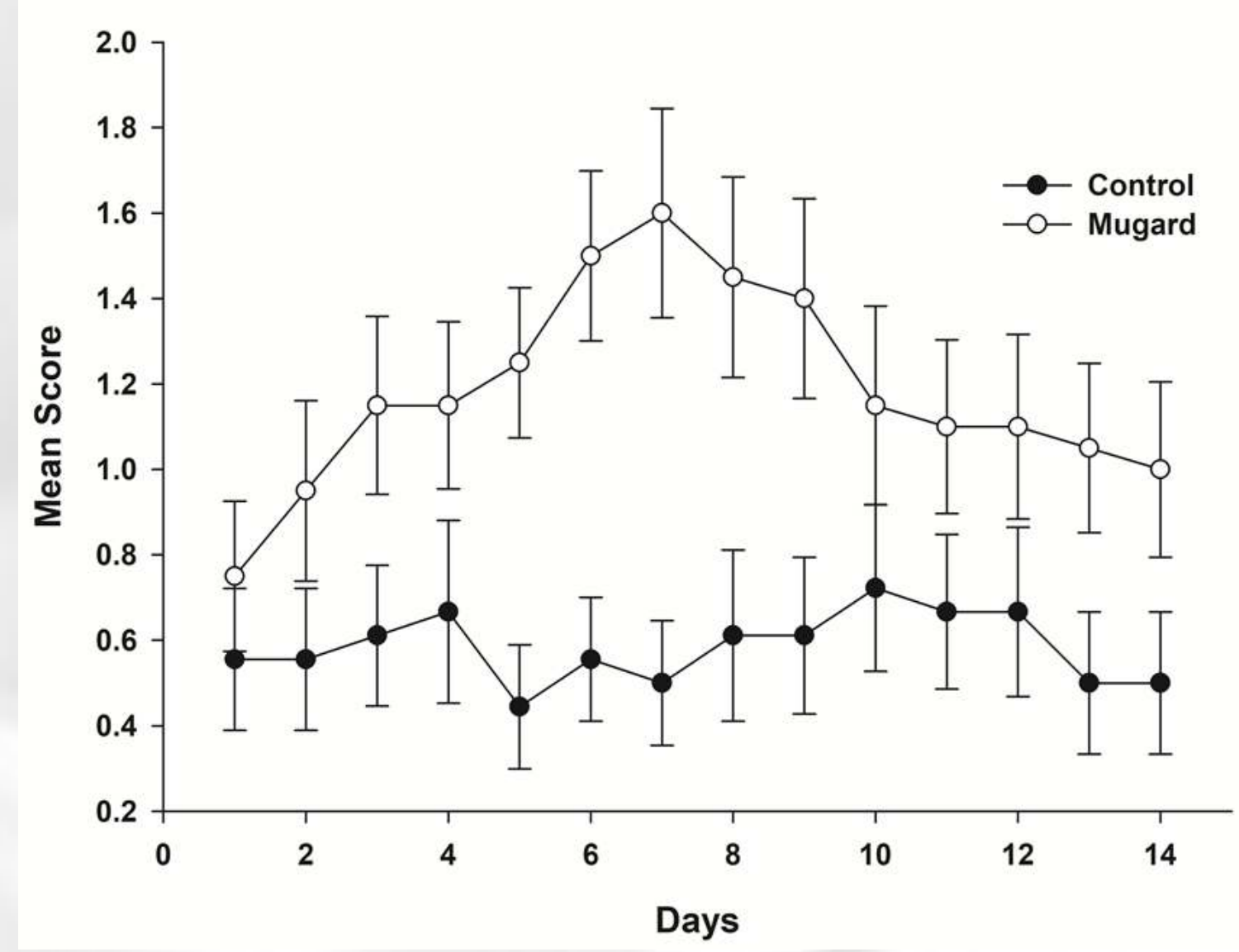

Of 49 patients enrolled. 38 patents (control, $N=18$; Mugard, $N=$ 20) were eligible for efficacy analysis. Recurrent rate of Mucositis grade $\geq 2$ was 2 patients $(11.1 \%)$ for control versus 5 patients $(25.0 \%)$ for MuGard ( $p=0.270)$. Time to first occurrence of Mucositis grade $>2$ was 4 days for control versus 7 days MuGard $(p=0.291)$. No significant adverse events were reported and the incidence of adverse events was not different between the MuGard group and the control saline group.

\section{CONCLUSION}

Although not statistically significant compared to saline, Mugard appears to have the effect of delaying the occurrence of severely graded ( $>2$ ) Mucositis when used in patients with a previous WHO grade 2 or higher mucositis. 